\title{
Spin manipulation and spin-lattice interaction in magnetic colloidal quantum dots
}

\author{
Fabrizio Moro, ${ }^{1, *}$ Lyudmila Turyanska, ${ }^{1}$ Josef Granwehr, ${ }^{2}$ and Amalia Patanè ${ }^{1}$ \\ ${ }^{1}$ School of Physics and Astronomy, The University of Nottingham, University Park, NG7 2RD, Nottingham, United Kingdom \\ ${ }^{2}$ Institute of Energy and Climate Research (IEK-9), Forschungszentrum Juelich, D-52425 Juelich, Germany
}

(Received 4 June 2014; revised manuscript received 10 October 2014; published 21 November 2014)

\begin{abstract}
We report on the spin-lattice interaction and coherent manipulation of electron spins in Mn-doped colloidal PbS quantum dots (QDs) by electron spin resonance. We show that the phase memory time, $T_{M}$, is limited by $\mathrm{Mn}-\mathrm{Mn}$ dipolar interactions, hyperfine interactions of the protons $\left({ }^{1} \mathrm{H}\right)$ on the QD capping ligands with Mn ions in their proximity $(<1 \mathrm{~nm})$, and surface phonons originating from thermal fluctuations of the capping ligands. In the low Mn concentration limit and at low temperature, we achieve a long phase memory time constant $T_{M} \sim 0.9 \mu \mathrm{s}$, thus enabling the observation of Rabi oscillations. Our findings suggest routes to the rational design of magnetic colloidal QDs with phase memory times exceeding the current limits of relevance for the implementation of QDs as qubits in quantum information processing.
\end{abstract}

DOI: 10.1103/PhysRevB.90.205428

PACS number(s): 75.50.Pp, 75.50.Tt, 76.30.Lh, 76.60.Lz

\section{INTRODUCTION}

In the last two decades the coherent manipulation of electron spins in semiconductor quantum dots (QDs) has attracted continuously increasing interest for quantum information processing (QIP) applications [1,2]. A basic requirement for QIP is that the phase memory time $\left(T_{M}\right)$ of the electron spin must be sufficiently long compared to the time required for computing [3]. Therefore an understanding of the sources of spin dephasing is necessary to design QDs with long $T_{M}$. Although spin manipulation has been reported for electron spins confined in lateral [4] and magnetic self-assembled [5] QDs, it still remains largely unexplored in colloidal QDs [6,7]. Nevertheless, significant advances in the synthesis of colloidal QDs have enabled the controlled doping of the QDs with magnetic impurities (e.g., Mn) [7,8], flexibility in manipulating the QD surface and environment [8,9], and implementation of the QDs in hybrid nanocomposite device structures [9]. Thus, colloidal magnetic semiconductor QDs represent an excellent benchmark not only to study the dephasing effects of electric (phonons) and magnetic (nuclei and unpaired electrons) field fluctuations on electron spin coherence but also to explore promising routes to quantum technologies.

Since the exchange of energy between the electron spin and the lattice unavoidably leads to spin dephasing, identifying the physical mechanisms responsible for the coupling between the electron spins and the phonon bath is crucial for achieving long phase memory times. The spin-lattice relaxation time constant, $T_{1}$, provides an upper bound for the coherence time constant $T_{2}$ (i.e., the lifetime for superposition of states) according to $T_{M}<T_{2} \leqslant 2 T_{1}$ [10]. Therefore, knowledge of $T_{1}$ provides the theoretical maximum value of the coherence time as well as the influence of spin-phonon interactions on the spin dynamics, which can take place either via one-phonon (direct) or twophonon (Raman or Orbach) processes [11]. To date, the role

\footnotetext{
${ }^{*}$ Corresponding author: fabrizio.moro@nottingham.ac.uk

Published by the American Physical Society under the terms of the Creative Commons Attribution 3.0 License. Further distribution of this work must maintain attribution to the author(s) and the published articles title, journal citation, and DOI.
}

played by the phonon bath on $T_{M}$ in colloidal QDs remains unknown. Understanding this important problem along with the ability to manipulate coherent states is of fundamental interest and essential for the design and utilization of QDs as qubits in future applications.

Here we report on the spin-lattice interaction and spin manipulation in Mn-doped PbS colloidal QDs by electron spin resonance (ESR). These narrow band gap semiconductor nanocrystals [12] may offer new opportunities for optical control of exciton qubits due to strong electron and hole confinement in the nanocrystal, optical emission tunable in the near-infrared wavelength region, controlled incorporation of $\mathrm{Mn}$ ions down to a few $\mathrm{Mn}$ atoms per QD [13], and compatibility with other low-dimensional semiconductors, such as graphene, to construct new hybrid functional devices [9]. Our ESR studies allow us to shed light on the dominant mechanism for spin-lattice relaxation and to identify the major sources of spin dephasing in (i) Mn-Mn dipolar interactions; (ii) hyperfine interactions of the protons $\left({ }^{1} \mathrm{H}\right)$ on the QD capping ligands with $\mathrm{Mn}$ ions in their proximity $(<1 \mathrm{~nm})$; and (iii) surface phonons originating from thermal fluctuations of the capping ligands. These phenomena could be observed in other nanocrystals and indicate that the control of the Mn content and position of the $\mathrm{Mn}$ ions within the nanocrystal and the conformational rigidity of the QD capping ligands are crucial for the rational design of QD qubits with $T_{M}$ exceeding the current limits.

\section{MATERIALS AND METHODS}

Colloidal Mn-doped $\mathrm{PbS}$ QDs with average diameter of $4.5 \pm 1.2 \mathrm{~nm}$ were synthesized in aqueous solution with Mn weight content, $x$, from $0.05 \%$ to $0.5 \%$, as detailed in Ref. [14], which corresponds to a statistical number of Mn ions per QD (Mn:QD) from 1:2 to 5:1 (Table I). Our approach enables the incorporation of $\mathrm{Mn}^{2+}$ ions into QDs and the controlled modification of their optical and magnetic properties. Solutions were freeze-dried to produce colloidal QDs powder samples for ESR studies.

Continuous-wave $(\mathrm{CW})$ and pulsed-ESR experiments were performed on a Bruker ElexSys E580 X-band spectrometer with a dielectric resonator (MD5). Continuous-wave ESR 
TABLE I. Estimates of the average number of Mn ions per QD (Mn:QD), average distance between Mn ions (d), magnetic dipolar field $\left(B_{\text {dip }}\right)$, dipolar frequency $\left(v_{\text {dip }}\right)$, and spin-spin dipolar relaxation time $\left(T_{\text {dip }}\right)$. For comparison, experimental values of $T_{1}, T_{\mathrm{SD}}$, and $T_{M}$ at $5 \mathrm{~K}$ and $B_{0}=345 \mathrm{mT}$ are also reported.

\begin{tabular}{|c|c|c|c|c|c|c|c|c|}
\hline$x \%$ & Mn:QD & $d(\mathrm{~nm})$ & $B_{\text {dip }}(\mu \mathrm{T})$ & $v_{\text {dip }}(\mathrm{MHz})$ & $T_{\mathrm{dip}}(\mu \mathrm{s})$ & $T_{\mathrm{M}}(\mu \mathrm{s})$ & $T_{1}(\mu \mathrm{s})$ & $T_{\mathrm{SD}}(\mu \mathrm{s})$ \\
\hline 0.05 & $1: 2$ & 5.8 & 46 & 1.3 & 0.8 & 0.83 & 160 & 30 \\
\hline 0.1 & $1: 1$ & 4.6 & 93 & 2.6 & 0.4 & 0.47 & 55 & 10 \\
\hline 0.3 & $3: 1$ & 3.2 & 280 & 7.8 & 0.1 & 0.10 & 14 & 2 \\
\hline 0.5 & $5: 1$ & 2.7 & 460 & 13.0 & 0.08 & 0.07 & 3 & 0.6 \\
\hline
\end{tabular}

experiments were performed with magnetic field modulation amplitude and frequency of $0.1 \mathrm{mT}$ and $100 \mathrm{kHz}$, respectively.

Echo field-swept (EFS) measurements were studied using a primary echo sequence, $\pi / 2-\tau-\pi-\tau-e c h o$, with $\pi=$ $32 \mathrm{~ns}, \tau=200 \mathrm{~ns}$, and shoots repetition time of $1048 \mu \mathrm{s}$. Echo decay traces were recorded by increasing the interpulse delay $\tau$ of the primary echo sequence [15]. $T_{M}$ was estimated by fitting the echo decay signal $(I)$ to the function:

$$
I(2 \tau)=I(0) \exp \left(-2 \tau / T_{M}\right) .
$$

Electron-nuclear hyperfine interactions cause a modulation of the primary echo amplitude, which represents a twopulse electron spin echo envelope modulation (2p-ESEEM) experiment. For a spin system with an electron spin $S=1 / 2$ and a nuclear spin $I=1 / 2$, the modulation can be described by the analytical equation [15]:

$$
\begin{aligned}
V_{2 p}(\tau)= & 1-\frac{k}{4}\left[2-2 \cos \left(\omega_{\alpha} \tau\right)-2 \cos \left(\omega_{\beta} \tau\right)\right. \\
& \left.+\cos \left(\omega_{-} \tau\right)+\cos \left(\omega_{+} \tau\right)\right],
\end{aligned}
$$

where $\omega_{\alpha}$ and $\omega_{\beta}$ are the nuclear frequencies for the two $m_{s}$ manifolds, $\omega_{+}=\omega_{\alpha}+\omega_{\beta}$ and $\omega_{-}=\omega_{\alpha}-\omega_{\beta}$ are the combination frequencies, and $k$ is the modulation depth parameter describing the amplitude of the oscillations. The parameter $k$ is a function of the angle $\theta$ between the direction of the applied static magnetic field, $\mathbf{B}_{0}$, and the interdistance vector, $\mathbf{r}$, between electron and nuclear spin.

Spin-lattice relaxation studies were carried out using an inversion recovery pulse sequence, $\pi-t-\pi / 2-\tau-\pi-$ $\tau-e c h o$, with $\tau=0.2 \mu$ s and variable $t$ [15]. Spin-lattice relaxation time constants were determined from a fit of the echo recovery amplitude to the biexponential function:

$$
I_{\text {echo }}(t)=I_{1} \exp \left(-t / T_{1}\right)+I_{\mathrm{SD}} \exp \left(-t / T_{\mathrm{SD}}\right),
$$

where $I_{1}$ and $I_{\mathrm{SD}}$ are amplitudes, and $T_{\mathrm{SD}}$ is the spectral diffusion time constant, which is known to affect the inversion recovery sequence [16]. In Sec. III, only the slower component $T_{1}$ is examined. Values of $T_{\mathrm{SD}}$ are listed in Table $\mathrm{I}$.

Electron spin nutation experiments were performed with the pulse sequence, $t_{p}-t-\pi / 2-\tau-\pi-\tau-e c h o$, where the nutation pulse length $t_{p}$ was varied, while $t=1 \mu \mathrm{s}$ and $\tau=0.2 \mu \mathrm{s}$.

\section{RESULTS AND DISCUSSION}

\section{A. Continuous-wave ESR}

Room temperature CW ESR spectra reveal six lines superimposed onto a broad signal (Fig. 1). By decreasing $x$, the resolution of the six lines increases, and at $x=0.05 \%$, additional weaker and narrower features are observed. The six line pattern is characteristic for isotropic hyperfine splitting in $\mathrm{Mn}^{2+}$ (with an electron spin $S=5 / 2$ and a nuclear spin $I=5 / 2$ ), thus indicating the incorporation of isolated $\mathrm{Mn}^{2+}$ ions into the nanocrystals. The sextet is centred close to the free electron $g$ value, $g_{e}=2.0025$, with a hyperfine constant $A \sim 9.3 \mathrm{mT}$. Similar values were reported for $\mathrm{PbS}: \mathrm{Mn}$ QDs in a glass matrix [17]. The six ESR lines are not exactly evenly spaced and are significantly broadened compared to those for $\mathrm{Mn}^{2+}$ in a hexagonal $\mathrm{ZnO}$ crystalline environment [18]. These results suggest that the local symmetry of $\mathrm{Mn}^{2+}$ ions is lower than cubic, as expected for ions located near or at the surface of a QD, which experience strain and crystal field anisotropies [17,19]. The additional broadening of the linewidth for samples with $x>0.1 \%$ is likely caused by increased dipolar interactions between $\mathrm{Mn}^{2+}$ ions [20,21].

\section{B. Mn concentration dependence of the electron spin dynamics and 2p-ESEEM}

At the maximum of the EFS intensity, $B_{0}=345 \mathrm{mT}$ (see Supplemental Material, S1 [22]), $T_{M}$ increases from $\sim 0.07 \mu \mathrm{s}$ to $\sim 0.8 \mu \mathrm{s}$ with $x$ decreasing from $0.5 \%$ to $0.05 \%$ [see Figs. 2(a) and 3(a)]. The longest $T_{M} \sim 0.9 \mu \mathrm{s}$ is achieved for $x=0.05 \%$ at $4.2 \mathrm{~K}$, which is up to two orders of magnitude longer than time constants previously reported for Mn-doped

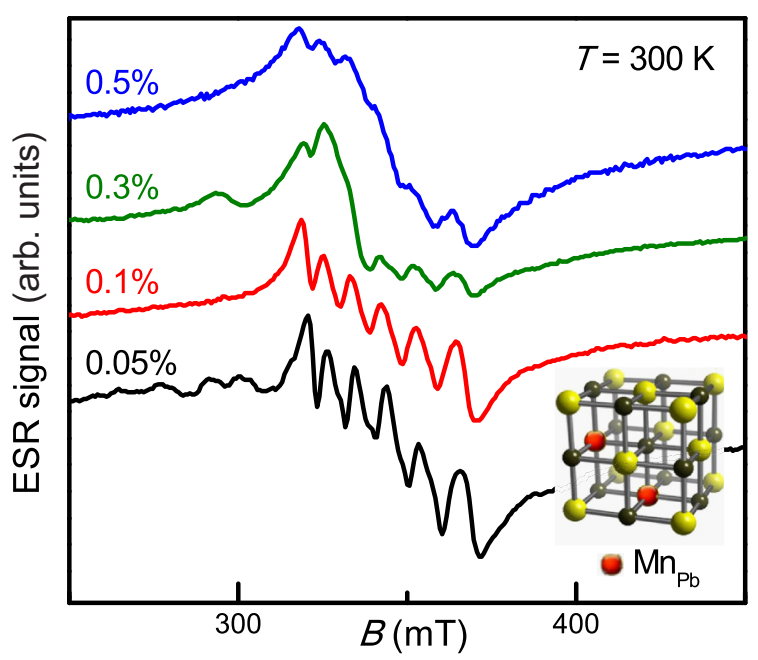

FIG. 1. (Color online) Continuous-wave ESR spectra of $\mathrm{PbS}: \mathrm{Mn}$ QDs at different Mn concentrations, $x$. Inset: sketch of the PbS:Mn crystal lattice with substitutional $\mathrm{Mn}_{\mathrm{Pb}}$. 

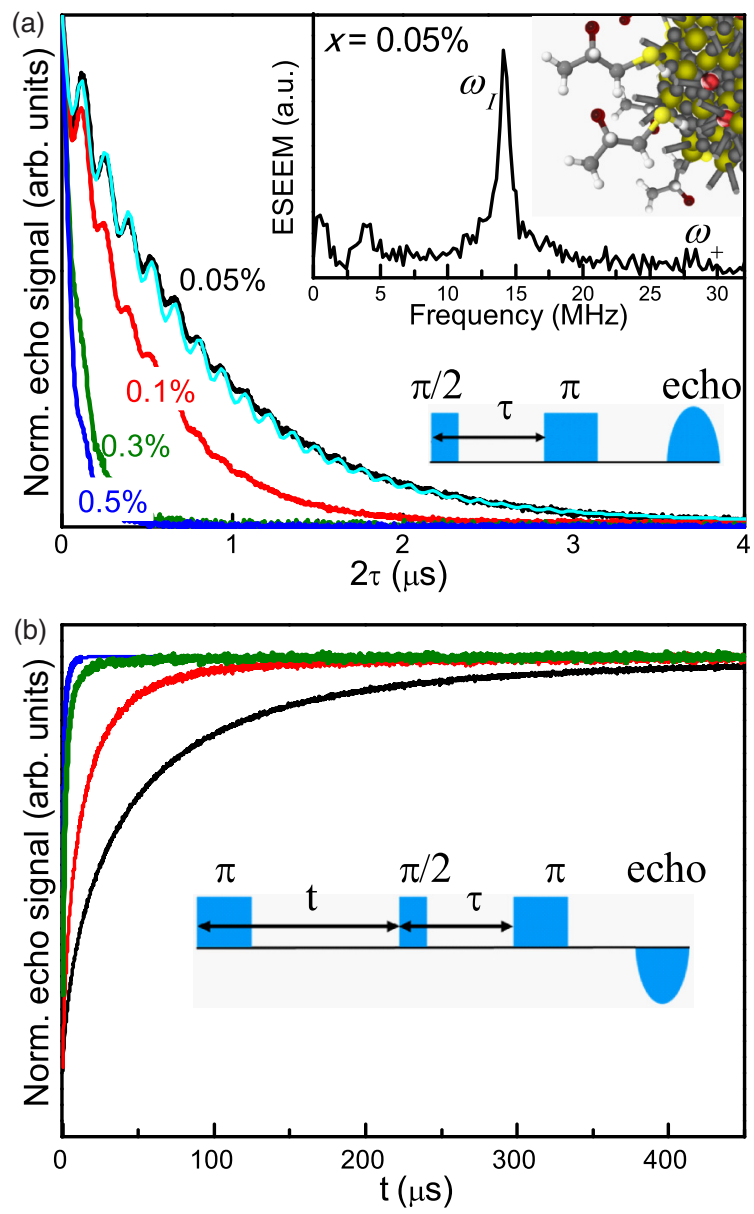

FIG. 2. (Color online) Spin echo (a) and inversion recovery (b) measurements at different $x$ at $5 \mathrm{~K}$. The cyan curve in (a) is the simulation to Eq. (4), as discussed in the text. Inset in (a) shows the ESEEM spectrum and cartoon of the PbS:Mn QDs. Pulse sequence schemes are also shown in (a) and (b).

CdTe semiconductor bulk layers [23] for $\mathrm{ZnSe} / \mathrm{MnSe}$ quantum wells [24] or for nonmagnetic In(Ga)As self-assembled QDs [25]. Our $T_{M}$ values are instead similar to those reported for Mn-doped $\mathrm{ZnO}$ colloidal QDs [6,7]. Also, we observe an ESEEM signal superimposed to the Hahn echo decay [Fig. 2(a)]. By subtracting the monoexponential decay and Fourier transforming the resulting curve, we extracted a characteristic modulating frequency at $14.3 \pm 0.5 \mathrm{MHz}$ [Fig. 2(a) inset]. This is close to the ${ }^{1} \mathrm{H}$ Larmor frequency $\left(\omega_{I} / 2 \pi=14.69 \mathrm{MHz}\right)$ and suggests coupling of electron spins to ${ }^{1} \mathrm{H}$ located in the QD-capping ligands. The weaker spectral features at $\sim 3.1 \mathrm{MHz}$ could be tentatively ascribed to interactions with ${ }^{207} \mathrm{~Pb}$ nuclei. A dominant contribution of ${ }^{1} \mathrm{H}$ nuclear spins to electron spin dephasing in comparison to other nuclear spins in the QD is ascribed to the larger gyromagnetic ratio $\left(\gamma_{1} \mathrm{H} / \gamma_{207} \mathrm{~Pb} \sim 5\right.$ and $\left.\gamma_{1} / \gamma^{33} \mathrm{~S} \sim 13\right)$ and natural abundance $\left(\sim 100 \%\right.$ for ${ }^{1} \mathrm{H}, \sim 22 \%$ for ${ }^{207} \mathrm{~Pb}$, and $\sim 0.8 \%$ for ${ }^{33} \mathrm{~S}$ ) of ${ }^{1} \mathrm{H}$.

Since the excitation of the ESR peak at $B_{0}=345 \mathrm{mT}$ mainly induces transitions between $m_{s}= \pm 1 / 2$ quantum numbers, we can treat our spin system as a fictitious $S^{\prime}=1 / 2$ and $I^{\prime}=1 / 2$ system. In this case a discussion of the ESEEM spectra can be based on Eq. (2). The unresolved $\omega_{\alpha}$ and $\omega_{\beta}$ peaks at $\sim 14.3 \mathrm{MHz}$ suggest that the isotropic electron $-{ }^{1} \mathrm{H}$ coupling is weak; therefore, we can analyze our data assuming that $\omega_{\alpha} \sim \omega_{\beta} \sim\left|\omega_{I}\right|$, and $\omega_{-} \sim 0$. The sum frequency of the ${ }^{1} \mathrm{H}$ signal, $\omega_{+}=2\left|\omega_{I}\right| \sim 29 \mathrm{MHz}$, is considerably weaker than expected from the analytical expression for 2p-ESEEM with ideal, nonselective pulses [15]. We ascribe this to the limited excitation bandwidth of the refocusing $\pi$ pulse $(\sim 31 \mathrm{MHz})$, which only partially excites $\omega_{+}$. Thus we do not consider the resonance at $\omega_{+}$in the analysis.

We use Eq. (2) to model the 2p-ESEEM data. For a sample with statistical distribution of $\theta$ and small modulation depth $k(k \ll 1)$, the $\tau$ dependence of the ESEEM signal can be approximated by the equation:

$$
I_{\mathrm{ESEEM}}(2 \tau) \approx I(0)\left[1+\langle k\rangle \cos \left(\omega_{I} \tau\right)\right] \exp \left(-2 \tau / T_{M}\right) .
$$

The observed mean modulation depth $\langle k\rangle[26]$ represents an average of $k(\theta)$ over the three-dimensional unit sphere:

$$
\langle k\rangle \approx\left\langle\left(\frac{3}{2} \frac{g \mu_{0} \mu_{B} \sin (2 \theta)}{4 \pi B_{0} r^{3}}\right)^{2}\right\rangle=\frac{6}{5}\left(\frac{g \mu_{0} \mu_{B}}{4 \pi B_{0} r^{3}}\right)^{2},
$$

where $r$ is the modulus of the electron-nucleus distance vector $\mathbf{r}, \mu_{0}$ is the vacuum permeability, and $\mu_{B}$ is the Bohr magneton. With these assumptions, a mean distance $\langle r\rangle$ between an electron and a nuclear spin from a statistical distribution of electron and nuclear spin pairs can be obtained:

$$
\langle r\rangle=\sqrt[6]{\frac{6}{5\langle k\rangle}} \sqrt[3]{\frac{g \mu_{0} \mu_{B}}{4 \pi B_{0}}} .
$$

The simulation of the echo decay for the sample with $x=$ $0.05 \%$ provides an estimate for $\langle k\rangle=0.08 \pm 0.01$, from which we derive $\langle r\rangle=0.28 \pm 0.03 \mathrm{~nm}$ by using Eq. (6). This value of $\langle r\rangle$ represents a lower limit for an electron spin interacting with a single proton. Assuming that all the coupled protons are at the same direction from the electron spin, which is justified by the small value of $\langle r\rangle$ compared to the averaged diameter of the QD, the integration of the product rule [26] for several nuclei provides the distance distribution of a single spin $S^{\prime}=1 / 2$ interacting with $n^{1} \mathrm{H}:\left\langle k_{n}\right\rangle=n\langle k\rangle$. Thus we calculate that even for $n=4$, the distance between electron and nuclear spins only increases to $\left\langle r_{4}\right\rangle=0.35 \mathrm{~nm}$ and for $n=20$ to $\left\langle r_{20}\right\rangle=0.46 \mathrm{~nm}$. These values are consistent with the assumption of weak electron $-{ }^{1} \mathrm{H}$ coupling, implying $\mathrm{Mn}$ ions close to the QD surface. For Mn ions located deeper in the core of QDs, the $\mathrm{Mn}-{ }^{1} \mathrm{H}$ interaction would be too weak to modulate the echo decay, while for $\mathrm{Mn}^{2+}$ ions at the surface and bound to the protons of the capping ligands, a nonvanishing isotropic hyperfine interaction would enable to resolve $\omega_{\alpha}$ and $\omega_{\beta}$ resonances.

The rate, $1 / T_{M}$, increases with $x$ [Fig. 3(a)], as expected from the gradual decrease of the average $\mathrm{Mn}-\mathrm{Mn}$ distance and corresponding enhancement of the magnetic dipolar field. This argument is supported by the broadening of the ESR linewidth with increasing $x$. For a spherical QD with average volume $V_{\mathrm{QD}}$, the number of Mn ions per QD is $N_{\mathrm{Mn}}=4 N_{\mathrm{uc}} x$, where $N_{\mathrm{uc}}=V_{\mathrm{QD}} / V_{\mathrm{uc}}$ is the number of PbS unit cells with volume $V_{\mathrm{uc}}=0.209 \mathrm{~nm}^{3}$ per QD and 4 is the number of $\mathrm{Pb}$ atoms per unit cell. Thus, the volume available for each $\mathrm{Mn}$ ion is 

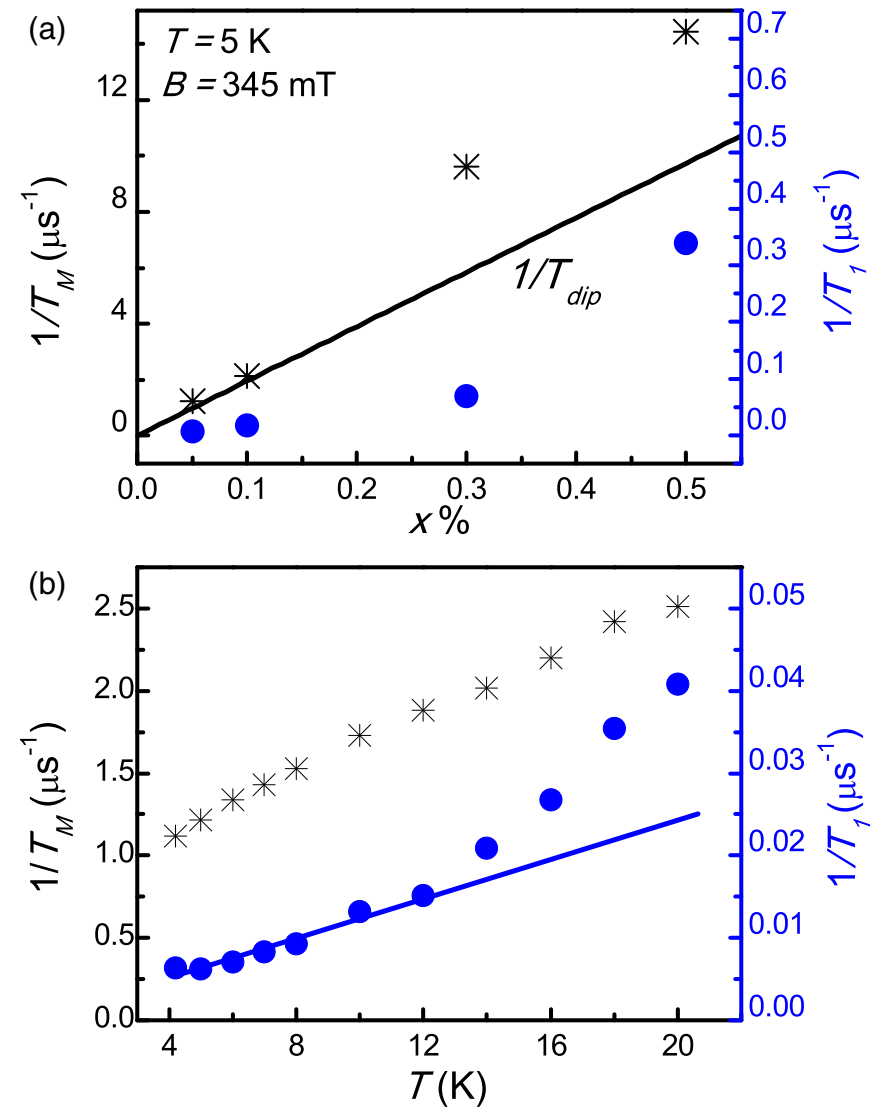

FIG. 3. (Color online) Dependence of $1 / T_{1}$ (circles) and $1 / T_{M}$ (stars) on the (a) Mn content and (b) temperature for $x=0.05 \%$. Experimental errors are within the size of the symbols. The line in (a) is the calculated dipolar limit, $1 / T_{\text {dip }}$. The line in (b) is a fit to $1 / T_{1}=A_{1} T$ with $A_{1}=(6.44 \pm 0.05) \times 10^{-3} \mu \mathrm{s}^{-1} \mathrm{~K}^{-1}$.

$V_{\mathrm{QD}} / N_{\mathrm{Mn}}$, and the average distance between $\mathrm{Mn}$ ions is $d=$ $\sqrt[3]{\frac{3}{2 \pi} \frac{V_{\mathrm{uc}}}{x}}$, which corresponds to the diameter of a sphere with volume $V_{\mathrm{QD}} / N_{\mathrm{Mn}}$. The results reported in Table I show that the $\mathrm{Mn}$ average distance increases from $\sim 2.7 \mathrm{~nm}$ to $\sim 5.8 \mathrm{~nm}$ for $x$ decreasing from $0.5 \%$ to $0.05 \%$, respectively.

From the classical dipolar field, $B_{\text {dip }}=\mu_{0}[3(\boldsymbol{\mu} \cdot \mathbf{d}) \mathbf{d}-$ $\left.\boldsymbol{\mu} d^{2}\right] / 4 \pi d^{5}$, where $\mathbf{d}$ is the interdistance $\mathrm{Mn}-\mathrm{Mn}$ vector and the resonance condition $B_{\text {dip }}=h v_{\text {dip }} / g \mu_{B}$, we estimate the lower limit for the decoherence rate, $v_{\text {dip }}$, set by the magnetic dipolar field, i.e., $v_{\text {dip }}=1 / T_{\text {dip }}=\mu_{0} g^{2} \mu_{B}^{2} S x / 3 h V_{\text {uc }}$, where $V_{\text {uc }}$ is the volume of a $\mathrm{PbS}$ unit cell and $S=5 / 2$ (Table I). We find that the values of $1 / T_{M}$ at different $x$ are close to the dipolar rate, $1 / T_{\text {dip }}$, and follow the expected linear dependence on $x$ [Fig. 3(a)]. Also, $1 / T_{M}$ does not reach a plateau at the lowest $x$, indicating that the dipolar coupling is a limiting factor for $T_{M}$.

The role played by phonons in the Mn-spin dynamics was inferred from the study of the spin lattice relaxation time constants, $T_{1}$ [Fig. 2(b)]. The rate of change of $1 / T_{1}$ as function of $x$ [Fig. 3(a)] flattens for $x<0.3 \%$ and increases at larger $x$. The coupling mechanisms responsible for the increase of $1 / T_{1}$ are magnetic dipolar interactions between neighboring magnetic ions and interactions of the magnetic ion with the crystal field [15]. Since the crystal field interacts directly only with the orbital motion of the electrons, the latter mechanism requires magnetic ions with orbital moment $L \neq 0$. On the other hand, we deduce that $L$ is quenched from the isotropic (nearly free) electron $g$ value (as found from the CW ESR studies) and the half-filled $d$ orbitals for $\mathrm{Mn}^{2+}$ ions in the ${ }^{6} \mathrm{~A}_{1}$ ground state [11]. Thus, we exclude this mechanism and notice instead that lattice waves could modulate the distance between nuclear or electron spins and, hence, spin-spin dipolar energies and anisotropic magnetic interactions. For a large Mn-Mn separation (i.e., $d>5 \mathrm{~nm}$ and $x<0.1 \%$ ), $1 / T_{1}$ is independent of $x$ [Fig. 3(a)]. For short Mn-Mn distances (i.e., $d<5 \mathrm{~nm}$ and $x>0.1 \%), 1 / T_{1}$ strongly depends on $x$, which is consistent with $\mathrm{Mn}-\mathrm{Mn}$ dipolar interactions modulated by lattice waves, thus shortening $T_{1}$.

\section{Temperature dependence of the electron spin dynamics}

The temperature variation of $1 / T_{M}$ [Fig. 3(b)] for $x=$ $0.05 \%$ is a clear manifestation of the link between $T_{2}$ (and hence $T_{M}$ ) and the lattice, which contributes to electron spin dephasing through thermal fluctuations of the surrounding spins. The weak and linear temperature dependence of $1 / T_{1}$ [Fig. 3(b)] indicates that the exchange of energy between the lattice and the Mn electron spin is mediated by one-phonon processes [11] at temperatures $T<10 \mathrm{~K}$. This spin-lattice interaction occurs only when the frequency of the lattice oscillations matches the Larmor frequency of the electron spins [15]. Because of the long phonon wavelength $(\lambda \sim 300 \mathrm{~nm}$ at $10 \mathrm{GHz})$ and small size of our nanocrystals $(d \sim 4.5 \mathrm{~nm})$, we ascribe lattice vibrations to surface phonons originating from fluctuations of capping ligands with low steric hindrance [27] rather than to QD core lattice vibrations [16]. For $T>10 \mathrm{~K}$, $1 / T_{1}$ shows a faster increase with temperature, suggesting that other relaxation mechanisms become dominant, e.g., two phonon Raman processes involving the excitation of virtual states [11]. The large energy separation $(\Delta \sim 2.4 \mathrm{eV})$ between the ground state $\left({ }^{6} \mathrm{~A}_{1}\right)$ and the excited state $\left({ }^{4} \mathrm{~T}_{1}\right)$ for $\mathrm{Mn}^{2+}$ in octahedral environment allows one to exclude Orbach processes involving the excitation of a state at energy $\Delta$ above the ground state [21]. We observe a similar temperature dependence of $1 / T_{1}$ and $1 / T_{M}$ for a sample with larger Mn content, $x=0.5 \%$ (see Supplemental Material, S2 [22]). This observation confirms that similar spin-lattice relaxation processes occur at different Mn concentrations, which we identify in the thermal motion of the capping ligands.

\section{Rabi oscillations}

The feasibility of quantum algorithms with spin-qubits depends on the number of driven coherent oscillations, as expressed by the figure of merit $Q_{M}=\Omega_{R} T_{M} / \pi$ [28], where $\Omega_{R}$ is the nutation (Rabi) frequency. Electron spin oscillations are shown in Fig. 4(a). We observe that the fast damping of the quantum oscillations is highly nonmonoexponential (see Supplemental Material, S3 [22]), likely due to an inhomogeneous $B_{1}$ across the sample. This can also be ascribed to a distribution of $\mathrm{Mn}$ electron spin coupling parameters due to strains caused by the proximity of the $\mathrm{Mn}^{2+}$ ions to the QD surface $[29,30]$ as well as to changes of the dipolar 

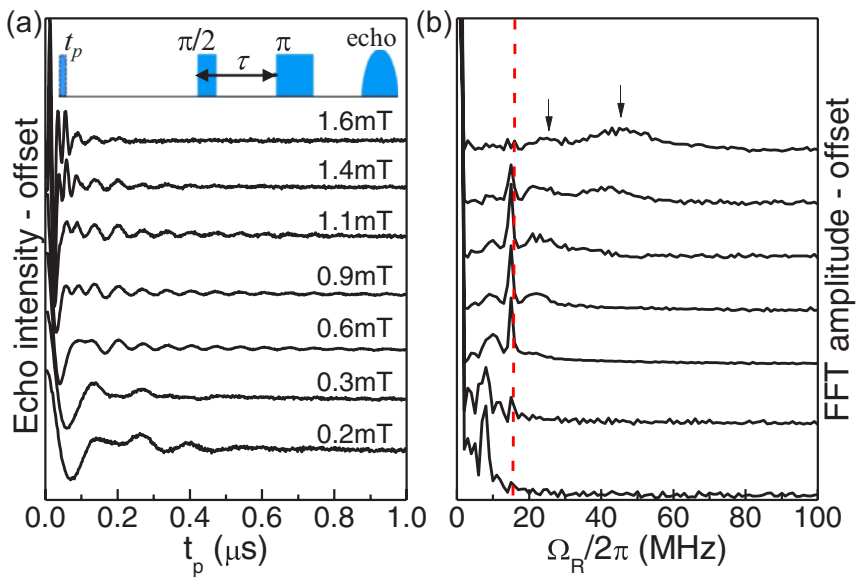

FIG. 4. (Color online) (a) Rabi oscillations and (b) fast Fourier transform for PbS:Mn QDs with $x=0.05 \%$ at various values of $B_{1}=0.2-1.6 \mathrm{mT}$ at $T=5 \mathrm{~K}$ and $B_{0}=345 \mathrm{mT}$. The nutation pulse scheme, the electron- ${ }^{1} \mathrm{H}$ nutation frequency (red dashed line) and the Rabi frequencies (arrows) are shown.

couplings occurring over the long nutation pulse [31]. The Fourier transform of the data recorded at different microwave powers $\left(P_{\mathrm{mw}} \propto B_{1}^{2}\right)$ show that the nutation frequency peaks shift from $\Omega_{R} / 2 \pi \sim 8 \mathrm{MHz}$ to $50 \mathrm{MHz}$ with increasing $B_{1}$ [Fig. 4(b)]. This demonstrates electron spin Rabi oscillations in a rotating frame of reference induced by the on-resonant irradiation of $B_{1}$ [15]. For $B_{1}=1.6 \mathrm{mT}$, we estimate a figure of merit $Q_{M} \sim 100$.

We note that the peak centered at $\sim 14.3 \mathrm{MHz}$ is independent of the microwave power [Fig. 4(b)], thus indicating that it originates from coherent electron- ${ }^{1} \mathrm{H}$ oscillations. These results support our 2p-ESEEM studies [Fig. 2(a) inset] and suggest that $\mathrm{Mn}$ ions are weakly coupled to ${ }^{1} \mathrm{H}$ spins on the QD surface. The intensity of the peak at $14.3 \mathrm{MHz}$ reaches the maximum value at $B_{1} \sim 0.6 \mathrm{mT}$, where the electron nutation frequency and Larmor frequency of ${ }^{1} \mathrm{H}$ spins are matched, i.e., both oscillate at the same frequency [32]. This condition also corresponds to the maximum number of quantum oscillations observed.
Our findings allow us to suggest the following design rules for the chemical engineering of QDs with longer $T_{M}$ and higher $Q_{M}$ : (i) overgrowing magnetically doped QDs with a diamagnetic shell to reduce hyperfine interactions between the $\mathrm{Mn}$ ions and the protons of the capping ligands; (ii) deprotonation of the capping ligands and of the solvent molecules could increase the $T_{M}$ value by up to a factor of 35 [16], in the limit of very high dilution where electron-electron dipolar interactions becomes negligible; (iii) the use of rigid capping ligands to minimize nuclear spin diffusion; and (iv) the dilution of the QDs in a diamagnetic matrix to minimize interparticle $\mathrm{Mn}-\mathrm{Mn}$ dipolar interactions.

\section{CONCLUSIONS}

We have reported on the spin-lattice interaction and spin manipulation by pulsed ESR of isolated, spatially confined $\mathrm{Mn}$ ions in $\mathrm{PbS}$ colloidal QDs and presented a protocol based on 2p-ESEEM to estimate the location of the Mn dopants. We found long phase memory times, which enabled us to coherently drive Rabi oscillations of Mn electron spins in the Bloch sphere. Moreover, we shed light on the dominant mechanisms responsible for spin-lattice interactions and identified the main sources of spin dephasing. A source of spin dephasing was found in surface phonons originating from fluctuations of the QD capping ligands. This phenomenon could be observed in other QDs and highlights the complexity of the mechanisms responsible for spin dephasing in nanostructures. Our findings allow us to propose the rational design of QD qubits with longer phase memory time constants. In addition, the large value of $T_{1}$ could lead to long exciton- and Mn-related optical emissions mediated by $s p-d$ interaction in the QDs [13], offering prospects to initialize, manipulate and read out spin-qubits [33,34].

\section{ACKNOWLEDGMENTS}

This paper is supported by The University Of Nottingham, The Leverhulme Trust, Grant No. RPG-2013-242, The Photon Science Institute and the Engineering and Physical Sciences Research Council (EPSRC) multifrequency Electron Paramagnetic Resonance (ESR) national facility at The University of Manchester. We are grateful to Dr. A. Fielding, Dr. W. Kockenberger, and Prof. N. R. Thomas for helpful discussions.
[1] R. Hanson and D. D. Awschalom, Nature 453, 1043 (2008).

[2] A. Greilich, D. R. Yakovlev, A. Shabaev, A. L. Efros, I. A. Yugova, R. Oulton, V. Stavarache, D. Reuter, A. Wieck, and M. Bayer, Science 313, 341 (2006).

[3] D. Loss and D. P. DiVincenzo, Phys. Rev. A 57, 120 (1998).

[4] J. R. Petta, A. C. Johnson, J. M. Taylor, E. A. Laird, A. Yacoby, M. D. Lukin, C. M. Marcus, M. P. Hanson, and A. C. Gossard, Science 309, 2180 (2005).

[5] L. Besombes, Y. Léger, L. Maingault, D. Ferrand, H. Mariette, and J. Cibert, Phys. Rev. Lett. 93, 207403 (2004).
[6] P. G. Baranov, S. B. Orlinskii, C. de Mello Donegá, and C. J. Schmidt, Phys. Status Solidi B 250, 2137 (2013).

[7] S. T. Ochsenbein and D. R. Gamelin, Nat. Nanotechnol. 6, 112 (2011).

[8] D. J. Norris, A. L. Efros, and S. C. Erwin, Science 319, 1776 (2008).

[9] J. H. Engel and A. P. Alivisatos, Chem. Mater. 26, 153 (2014).

[10] V. N. Golovach, A. Khaetskii, and D. Loss, Phys. Rev. Lett. 93, 016601 (2004).

[11] A. Abragam and B. Bleaney, Electron Paramagnetic Resonance of Transition Ions (Oxford University Press, Oxford, 1970).

[12] M. A. Hines and G. D. Scholes, Adv. Mat. 15, 1844 (2003). 
[13] L. Turyanska, R. J. A. Hill, O. Makarovsky, F. Moro, A. N. Knott, O. J. Larkin, A. Patanè, A. Meaney, P. C. M. Christianen, M. W. Fay, and R. J. Curry, Nanoscale 6, 8919 (2014).

[14] L. Turyanska, F. Moro, A. N. Knott, M. W. Fay, T. D. Bradshaw, and A. Patanè, Part. Part. Syst. Char. 30, 945 (2013).

[15] A. Schweiger and G. Jeschke, Principles of Pulse Electron Paramagnetic Resonance (Oxford University Press, Oxford, 2005).

[16] S. S. Eaton and G. R. Eaton, Relaxation Times of Organic Radicals and Transition Metal Ions (Springer-Verlag, Berlin, 2000).

[17] R. S. Silva, P. C. Morais, F. Qu, A. M. Alcalde, N. O. Dantas, and H. S. L. Sullasi, Appl. Phys. Lett. 90, 253114 (2007).

[18] A. Hausmann and H. Huppertz, J. Phys. Chem. Solids 29, 1369 (1968).

[19] N. S. Norberg, K. R. Kittilstved, J. E. Amonette, R. K. Kukkadapu, D. A. Schwartz, and D. R. Gamelin, J. Am. Chem. Soc. 126, 9387 (2004).

[20] P. H. Borse, D. Srinivas, R. F. Shinde, S. K. Date, W. Vogel, and S. K. Kulkarni, Phys. Rev. B 60, 8659 (1999).

[21] W. Chen, R. Sammynaiken, Y. N. Huang, J. O. Malm, R. Wallenberg, J. O. Bovin, V. Zwiller, and N. A. Kotov, J. Appl. Phys. 89, 1120 (2001).

[22] See Supplemental Material at http://link.aps.org/supplemental/ 10.1103/PhysRevB.90.205428 for EFS spectra and their numerically calculated first derivatives for $\mathrm{PbS}: \mathrm{Mn}$ with different concentrations of Mn ions (S1), temperature dependence of the
$T_{1}$ and $T_{m}$ relaxation rates for PbS:Mn with $x=0.5 \%$ and $0.05 \%$ (S2), and simulation of a nutation experiment (S3).

[23] T. Dietl, P. Peyla, W. Grieshaber, and Y. M. Daubigne, Phys. Rev. Lett. 74, 474 (1995).

[24] S. A. Crooker, D. A. Tulchinsky, J. Levy, D. D. Awschalom, R. Garcia, and N. Samarth, Phys. Rev. Lett. 75, 505 (1995).

[25] R. J. Warburton, Nat. Mater. 12, 483 (2013).

[26] W. B. Mims, J. L. Davis, and J. Peisach, J. Magn. Reson. 86, 273 (1990).

[27] A. Zecevic, G. R. Eaton, S. S. Eaton, and M. Lindgren, Mol. Phys. 95, 1255 (1998).

[28] S. Bertaina, S. Gambarelli, A. Tkachuk, I. N. Kurkin, B. Malkin, A. Stepanov, and B. Barbara, Nat. Nanotechnol. 2, 39 (2007).

[29] S. Bertaina, S. Gambarelli, T. Mitra, B. Tsukerblat, A. Müller, and B. Barbara, Nature 453, 203 (2008).

[30] J. Granwehr, A new approach to longitudinally detected electron paramagnetic resonance, Ph.D. thesis (http://dx.doi.org/10.3929/ethz-a-004320800) ETH (2002).

[31] K. Benzid, A. Chetoui, M. Maamache, P. Turek, and J. Tribollet, Europhys. Lett. 104, 47005 (2013).

[32] G. Jeschke and A. Schweiger, J. Chem. Phys. 105, 2199 (1996).

[33] C. Le Gall, R. S. Kolodka, C. L. Cao, H. Boukari, H. Mariette, J. Fernández-Rossier, and L. Besombes, Phys. Rev. B 81, 245315 (2010).

[34] K. De Greve, D. Press, P. L. McMahon, and Y. Yamamoto, Rep. Prog. Phys. 76, 092501 (2013). 